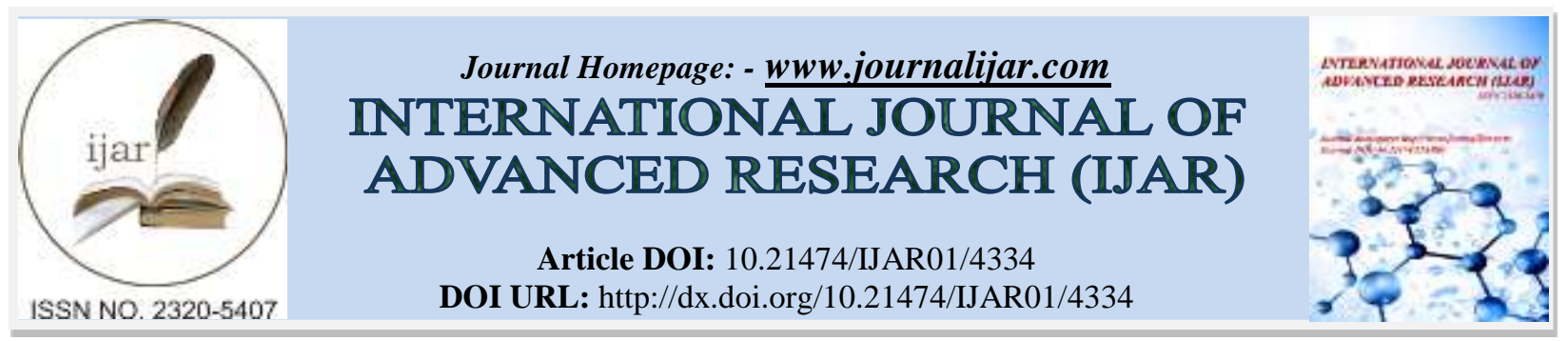

RESEARCH ARTICLE

\title{
PHYSICAL THERAPY MANAGEMENT IN MYOFASCIAL PAIN SYNDROME OF SHOULDER COMPLEX.
}

\author{
Shwetha $\mathbf{S}^{1}$, Hrishikesh $\mathrm{K}^{2}$, Ranjith $\mathrm{A}^{2}$, Komal $\mathbf{M}^{2}$, Naga Sumanth $\mathrm{G}^{3}$ and Sateesh $\mathrm{K}^{4}$. \\ 1. BPT, Srinivas College of Physiotherapy and Research Centre,Mangalore,575001,India. \\ 2. Assistant Professor, Srinivas College of Physiotherapy and Research Centre,Mangalore,575001,India. \\ 3. Head of the Department,Yashoda Hospitals,Hyderabad,500003,India ${ }^{4}$ MPT, Srinivas College of Physiotherapy \\ and Research Centre,Mangalore,575001,India.
}

\section{Manuscript Info}

n.......................

Manuscript History

Received: 22 March 2017

Final Accepted: 23 April 2017

Published: May 2017

Key words:-

Physiotherapy Management, Myofascial

Pain Syndrome, Shoulder Complex,

Manual Therapy

\section{Abstract}

Objective: To review the existing literature on myofascial pain syndrome of shoulder complex and imply the importance of physiotherapy in management in it.

Method: Search strategies from databases like Google scholar, PubMed, PubMed central, Scopus Index, Cochrane library and cross references of retrieved articles. Full text and abstract articles from the year 2002-2016 were included relating to myofascial pain syndrome and physical therapy management in shoulder complex.

Discussion: Various Types Of Physical Therapy Managements Such As Dry Needling, Myofascial Release, Fascial Manipulation, Facilitated Positional Release, Muscle Energy Technique, Kinesiotaping, Low-Level Laser Therapy, Cognitive Behavioral Therapy Are Effective In Treating Myofascial Pain Syndrome Of Shoulder Complex In Terms Of Reducing Pain, Improving Range Of Motion And Improving Functional Capacity.

Conclusion: Untreated and undiagnosed myofascial pain syndrome may develop psychological and functional problems, its early diagnosis and treatment may help to reduce overlying psychological complications and financial burden. In spite all these treatment still better treatment protocol is required to treating myofascial pain syndrome. The proper treatment of myofascial pain syndrome may be one of the most rewarding if handled correctly.

Copy Right, IJAR, 2016,. All rights reserved.

\section{Introduction:-}

The word "myofascia" is derived from the Greek word "myo", which means "muscle" and the word "fascia" refers to the protective covering surrounding a muscle ${ }^{1}$. Myofascial pain syndrome (MPS) is defined as musculoskeletal pain condition characterized by local and referred pain perceived as deep and aching, and by the presence of myofascial trigger point in any part of the body ${ }^{2}$. MPS is characterized by Myofascial Trigger Points (MTrPs). A trigger point can be located in fascia, ligaments, muscles, and tendons; however, MTrPs are also found in skeletal muscles and or their fascia. An MTrPs is a hyperirritable spot, associated with a taut band of a skeletal muscle that painful on compression or stretch, and that can give rise to a typical referred pain pattern as well as autonomic phenomena ${ }^{3}$.There are many theories available supporting evidence of the existence of MTrPs. ${ }^{4,5,6}$ Simons has 
recently proposed a new "integrated trigger point hypothesis" 7 . The integrated trigger point hypothesis has evolved through several steps of progress since its first introduction as the "energy crisis hypothesis" in 1981. The hypothesis builds on the finding that excessive amounts of acetylcholine from the motor nerve terminal cause miniature motor endplates potentials that produce the endplate noise observed with needle EMG of MTrPs. The excessive acetylcholine maintains a sustained depolarization of the post junctional membrane, which in turn results in an excessive release of calcium from the sarcoplasmic reticulum and sustained sarcomeres' contractions. Studies also suggested that an impaired re-uptake of calcium into the sarcoplasmic reticulum induced by calcium channel blockers may cause MTrPs ${ }^{8,9}$.
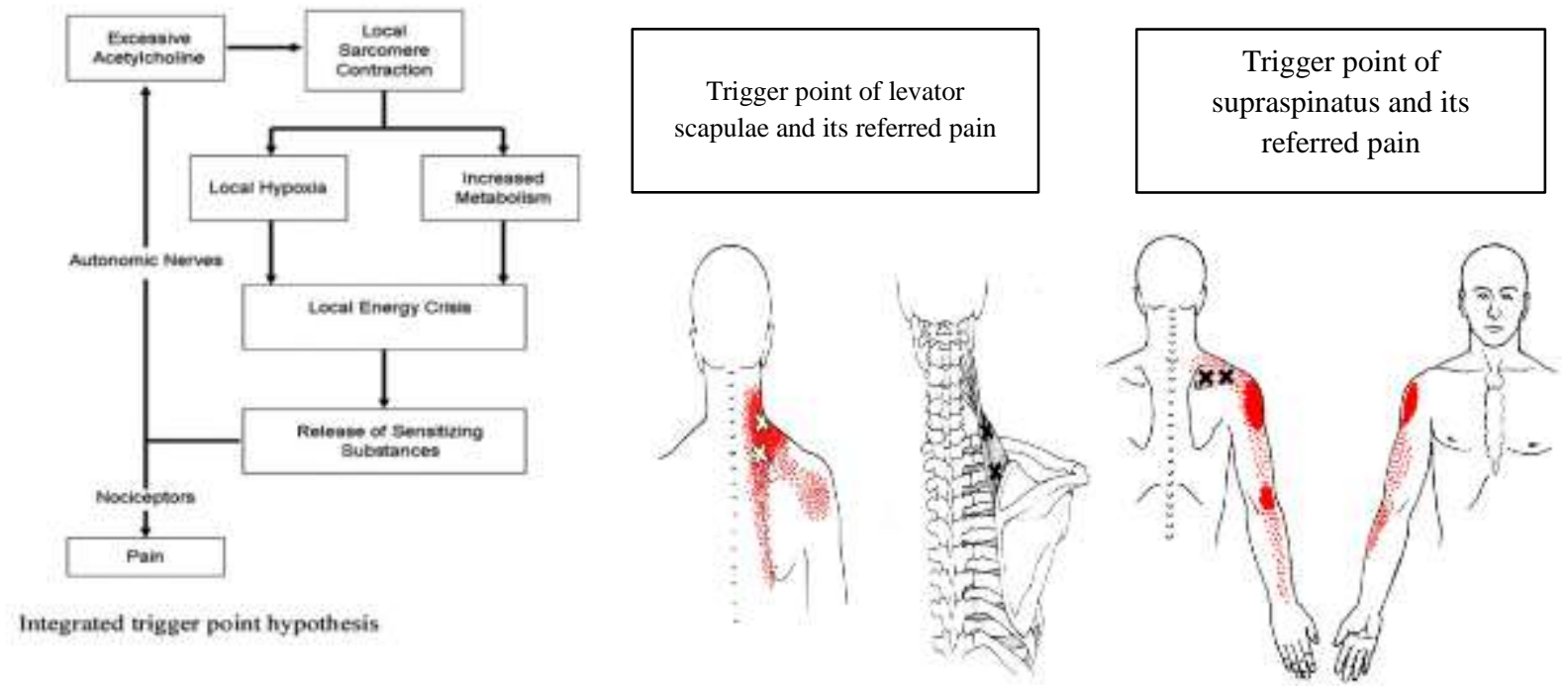

\section{Incidence:-}

Epidemiological data indicate the frequent incidence of the Active MTrPs were most prevalent in the infraspinatus (77\%) and the upper trapezius muscles (58\%), whereas latent MTrPs were most prevalent in the teres major (49\%) and anterior deltoid muscles (38\%). The number of muscles with active MTrPs was only moderately correlated with the DASH score ${ }^{10}$. Myofascial pain syndrome is diagnosed in $37 \%$ of men and $60 \%$ of women aged 30-60 years. ${ }^{5}$ Occupational neck and shoulder pain is more common in women than in men. Pressure pain thresholds (PPT) are also lower for women, signifying greater hypersensitivity to mechanical stimulation ${ }^{11}$. Researchers established an immediate drop in the concentrations of several substances with dry needling of an MTrPs. If the integrated trigger point hypothesis is basically correct, MTrPs are primarily a muscle disease with but important sensory, motor and autonomic phenomena. ${ }^{9,12}$

\section{Risk factors:-}

Patients with chronic myofascial pain often have problems that predispose those developing MPS. These factors need to be identified and treated where appropriate and possible. Ergonomic factors ${ }^{13,14}$. Hypermobility, Forward Neck Posture and Prolonged maintenance of posture may have the same effect as repeated low-level muscle activation. Involvement of work related muscle overload has been extensively studied. Physiological factors like Fatigue, general fitness, conditioning and coordination, Iron and Vitamin D deficiency have contributions in Myofascial pain syndrome of shoulder ${ }^{15}$.

\section{Clinical features:-}

A common symptom of active myofascial trigger points where the patient may complain of a pain ranging from a mild ache to an excruciating pain are either sharp or dull and is often associated with general fatigue and a decreased range of motion and loss of muscle strength. Myofascial pain is often referred to a distant site from the MTrPs, in a characteristic pattern for that muscle and sometimes patients are even aware of a numbness or paresthesia rather than pain. Patients often complain of disturbed sleep as a result of myofascial pain syndrome, which can lead to a vicious cycle of increased pain sensitivity the following day ${ }^{16,17}$.

Common signs of active myofascial trigger points where Studies concluded that all 5 major Criteria should be present and at least 1 of the minor criteria ${ }^{3,4,13}$. Major criteria includes Regional pain complaint, Pain pattern follows 
a known distribution of muscular referred pain, Palpable taut band (inaccessible muscles), Exquisite focal tenderness at one point or module within a taut band, Some restricted range of motion or muscle weakness (when measurable) and Minor criteria includes Manual pressure on the MFTP module reproduces the chief pain complaint, Snapping palpation of the taut band at the MFTP elicits a local twitch response, Pain is diminished or eliminated by muscular treatment, e.g. therapeutic stretch, ischemic compression or needle injection of the MFTP.

\section{Common Shoulder Pain Patterns:-}

Active TP's in the scalene muscles may refer pain in three directions, anteriorly with two finger- like projections to the anterior chest, down the arm to the fingers, and posteriorly to the upper back over the scapular attachment of the levator scapulae. Active TP's in the scalene anterior and medius may refer pain to the thumb and index finger." Additional entrapment symptoms may be caused by TP shortening of the scalene muscles ${ }^{7}$.Ten muscles can cause anterior shoulder pain: anterior deltoid, infraspinatus, biceps brachii, brachialis, scaleni, pectoralis major and minor, sternalis, latissimus dorsi (rare), and coracobrachialis. Three muscles can cause middle deltoid pain: middle deltoid, teres minor, and supraspinatus. Seven muscles can refer pain to the posterior shoulder and nearby scapular region: teres major, sub scapularis, rhomboids, serratus posterior superior, triceps brachii, latissimus dorsi, and levator scapulae?

\section{Diagnosis:-}

The diagnostics of the myofascial pain syndrome should include a detailed analysis of a patient's pain history with a clinical examination. The examination should include precise noting of pain areas (indicated by the patient) combined with a palpation, which allows for establishing the patterns of pain radiating from individual muscles ${ }^{5}$. Information helpful in diagnostics may be pain caused during palpation (recognized by the patient), tender point felt within the taut band of muscle fibers, local twitch response, "jump sign", weakening of muscular strength, restricted range of motion and pain resulting from stretching the muscle. The main diagnostic criteria are finding a taut band of muscle fibers and locating a tender, "nodular" area in muscle ${ }^{18}$.

\section{Physiotherapy Management:-}

Treatment of myofascial pain requires the inactivation of MTrPs, the restoration of normal muscle length, and the elimination or correction of the factors that created or perpetuated the trigger points in the first place. Many patients with chronic pain develop kinesophobia or fear-avoidance ${ }^{10}$. Physical therapy plays an important role in overcoming kinesophobia and facilitating active movement. Aggressive mobilizations and strengthening programs should be avoided as they may contribute to the development and maintenance of kinesiophobia ${ }^{19}$.

\section{Dry Needling:-}

Dry Needling is a skilled intervention that uses a thin filiform needle to penetrate the skin and stimulate underlying myofascial trigger points, muscular, and connective tissues for the management of neuromusculoskeletal pain and movement impairments ${ }^{20,21,22}$. Deep DN has been shown to inactivate MTrPs by eliciting local twitch responses $(\text { LTR })^{23}$. Superficial dry needling, which involves placing an acupuncture needle in the skin overlying an MTrPs and electrical twitch-obtaining intramuscular stimulation ${ }^{21,24}$. Based on previous infraspinatus studies, Trp-DN produces segmental anti nociceptive effects. On the one hand, active MTrPs-DN improves shoulder pain and the irritability of the satellite MTrPs in the referred pain area ${ }^{8,25}$. For patients with myofascial pain syndrome of the infraspinatus muscle and shoulder pain, dry needling of trigger points of that muscle showed an immediate reduction of pain and improvement of range of movement ${ }^{26}$.

\section{Myofascial Release:-}

Myofascial release is a collection of techniques used for the purpose of relieving soft tissue from an abnormal hold of a tight fascia. MFR treatment restores muscle function, promote relaxation and self-healing. There is no special test for this technique, but we can always measure ROM, mark pain scale, test strength before and after the treatment $^{27,28,29}$. They commonly classified as Direct Myofascial Release, Indirect Myofascial Release, and SelfMyofascial Release.

\section{Fascial Manipulation:-}

The manual therapy technique presents a biomechanical model to decipher the role of fascia in musculoskeletal disorders considering that the myofascial system is a three-dimensional continuum. In Facial Manipulation, the body is divided into 14 segments: head, neck, thorax, lumbar, pelvis, scapula, humerus, elbow, carpus, digits, hip, knee, ankle, and foot. Each body segment is served by six myofascial units (of units) consisting of mono articular and bi 
articular unidirectional muscle fibers, their deep fascia and the articulation that they move in one direction on one plane. The center of coordination is identified within each unit. Each center of coordination is located in the point of convergence of the vectored muscular forces that act on a body segment during a precise movement. The manual technique itself consists in creating localized heat by friction by using the elbow, knuckle, or fingertips on the above-mentioned points. ${ }^{30,31}$

\section{Facilitated Positional Release:-}

PRT originally termed strain-counter strain is a therapeutic technique that uses tender points (TPs) and a position of comfort (POC) to resolve the associated dysfunction ${ }^{32}$.

\section{Muscle Energy Technique:-}

Muscle energy techniques (MET) are a class of soft tissue osteopathic manipulation methods that incorporate precisely directed and controlled patient initiated, isometric contractions, designed to improve musculoskeletal function and reduce pain33. The MET has an effect in reducing pain, increase ROM and functional ability in the patient with adhesive Capsulitis ${ }^{33,34}$. Evidence suggest MET (OR similar isometric stretching techniques) is more effective than passive stretching for increasing muscle extensibility. ${ }^{34,35,36}$

\section{Kinesiotaping:-}

Kinesio-taping has four effects, to normalize muscular function, to increase lymphatic and vascular flow, to diminish pain and aid in the correction of possible articular misalignments. The myofascial pain of the deltoid muscle has treated an application of Kinesio- taping for the deltoid, reinforced by a transverse strip over the region where the MTPs are located. ${ }^{37,38,39}$

\section{Low-Level Laser Therapy (Lllt):-}

Laser therapy is effective in the treatment of musculoskeletal disorders through its analgesic, myo-relaxant, and tissue healing and bio-stimulation effects. Electronic Digital Algometer, "force one gauge-model FDI" was used to detect and confirm the site and sensitivity of trigger points by determining the (PPT) using a pressure transducer probe. ${ }^{40,41 .}$

\section{Cognitive Behavioural Therapy:-}

Reinforce wellness behaviors such as increased activity or participation in an exercise program. Relaxation therapies include a number of strategies aimed at lowering general arousal and promoting a state of relaxation and include biofeedback, imagery, diaphragmatic breathing, autogenic training, and progressive muscle relaxation training. ${ }^{42,43,44}$

\section{Conclusion:-}

Myofascial pain syndrome of shoulder is a common and treatable cause of morbidity. If left undiagnosed and untreated, it may develop into chronic pain with overlying psychosocial and functional problems. This may lead to further distress, anxiety and even depression. This major source of musculoskeletal dysfunction requires more focused attention. Its early diagnosis and treatment may help to reduce overlying psychosocial complications and the attending financial burden of chronic pain syndrome.Surgical management should be considered only after reasonable nonsurgical efforts have been tried. In spite of all above efforts, more research is still required for the better management of the patient suffering from this syndrome. The proper treatment of Myofascial Pain Syndrome may be one of the most rewarding if handled correctly.

\section{Reference:-}

1. Dorsher PT. Trigger points and myofascial pain. Myofascial Trigger Points. 2013; 41-8.

2. Saavedra FJCA, Cordeiro MT, Alves JV, Fernandes HM, Reis VM, Mont'alverni DGB. The influence of positional release therapy on the myofascial tension of the upper trapezius muscle. Revista Brasileira de Cineantropometria e Desempenho Humano. 2014; 16(2).

3. Cesar Fernandez de las Penas, Campo MS, Carnero JF, Page JCM. Manual therapies in myofascial trigger point treatment: a systematic review. Journal of Bodywork and Movement Therapies. 2005; 9(1):27-34.

4. Aguilera FJMCB, Martín DP, Masanet RA, Botella AC, Soler LB, Morell FB. Immediate Effect of Ultrasound and Ischemic Compression Techniques for the Treatment of Trapezius Latent Myofascial Trigger Points in Healthy Subjects: A Randomized Controlled Study. Journal of Manipulative and Physiological Therapeutics. 2009; 32(7):515-20. 
5. Chochowska MC, Szostak L, Marcinkowski J, Jutrzenka-Jesion J. Differential diagnosis between fibromyalgia syndrome and myofascial pain syndrome. Journal of Pre-Clinical and Clinical Research. 2015;9(1):82-6.

6. Schwartz JCI. Chronic Refractory Myofascial Pain: Characteristics of Patients who Self-select Long-term Management with Electrical Twitch-Obtaining Intramuscular Stimulation. International Journal of Physical Medicine \& Rehabilitation. 2013;01(04).

7. Nielsen AJ. Case Study: Myofascial Pain of the Posterior Shoulder Relieved by Spray and Stretch. Journal of Orthopaedic \& Sports Physical Therapy. 1981;3(1):21-6.

8. Calvo-Lobo CCA, Pacheco-Da-Costa S, Martínez-Martínez J, Rodríguez-Sanz D, Cuesta-Álvaro P, LópezLópez D. Dry Needling on the Infraspinatus Latent and Active Myofascial Trigger Points in Older Adults With Nonspecific Shoulder Pain. Journal of Geriatric Physical Therapy. 2016;:1.

9. Dommerholt J. Myofascial Pain Syndrome-Trigger Points. Journal of Musculoskeletal Pain. 2009Dec;17(4):423-31.

10. Carel Bron, Jan Dommerholt, Boudewijn Stegenga, Michel Wensing and Rob AB Oostendorp. High prevalence of shoulder girdle muscles with myofascial trigger points in patients with shoulder pain; Bron et al. BMC Musculoskeletal Disorders 2011, 12:139.

11. Matteo Castaldo, Hong-You Ge, Alessandro Chiarotto, Jorge H. Villafane et al, Med. Sci. Myofascial Trigger Points in Patients with Whiplash-Associated Disorders and Mechanical neck pain; Pain Medicine 2014;Wiley Periodicals, Inc.

12. Jari Y. Pressure algometry. The Australian journal of physiotherapy. 2007, Feb; 53.

13. Robert D G. Muscle Pain Diagnosis and Treatment; Chapter; Myofascia pain syndrome; 2010; 28-33.

14. Eng-Ching Yap. Myofascial Pain - An Overview; Ann Acad Med Singapore 2007; 36:43-8.

15. Robert D Gerwin. A review of myofascial pain and fibromyalgia- factors that promote their persistence; Acupuncture in Medicine; 2005; 23(3):121-134.

16. Paul J. Christo \& Kai McGreevy. Updated Perspectives on Neurogenic Thoracic Outlet Syndrome; Curr Pain Headache Rep; 23 November 2010.

17. Jacob Isaac Jaso, Ganesh Sundaram S, Vengata Subramani M. Physiotherapy Intervention For Adhesive Capsulitis of Shoulder: A Systematic Review; Int J Physiother Res 2015, 3(6):1318-25.

18. Robert D. Gerwin, Jan Dommerholt and Jay P. Shah. Current Pain and Headache Reports. 2004, 8:468-475.

19. Jan Dommerholt. Complex regional pain syndrome: physical therapy management; Journal of Bodywork and Movement Therapies 2004, 8; 241-248.

20. Karel Lewit, and Sarka Olsanska. Clinical Importance of Active Importance of Active Scars as a Cause of Myofascial; J Manipulative PhysioTher 2004; 27:399-402

21. Tamer S. Issa, Peter A. Huijbregts. Physical Therapy Diagnosis and Management of a Patient with Chronic Daily Headache: A Case Report; The Journal of Manual \& Manipulative Therapy; 2006,14; 88 - 123.

22. Marek Wyszynski.The New York Pain Treatment Program Protocol A Structured Physical Therapy Approach for Treating the Muscular Components of Chronic Pain Syndromes; 2002.

23. Edward S.Rachlin. Myofascial Pain and Fibromyalgia; Trigger point Management;1994;143-175.

24. Tubbs, R. Shane, Tyler-Kabara, Elizabeth C, Aikens Alan C, Martin Justin P.Weed Leslie L. Salter E. George and Oaks Jerry.Surgical Anatomy of the dorsal scapular nerve;J.Nerurosurg 2005,105;910-911.

25. Jhonson McEvoy, Jan Dommerholt.Physical Therapy Of Shoulder;Fifth Edition;Chapter 16 Myofascial Trigger Points Of The Shoulder; Elseivier Churchill Livingstone;2012;354-356.

26. Rached RA, Rampim DB, Yamauti RH, Azeka MS, Santos RM, Guidolin B, et al.Rotator cuff syndrome: rehabilitation; Acta Fisiatr. 2013; 20(2):96-105.

27. Nizar abd Jalil, Mohammad Saufia Wang, Mahamarowi omar. Scalene Myofascial Pain Syndrome Mimicking Cervical Disc Prolapse: A Report of Two Cases; Malaysian J Med Sci. Jan-Mar 2010; 17(1): 60-66.

28. Salvi Shah, Akta Bhalara. Myofascial Release; International Journal of Health Sciences \& Research Vol.2; Issue: 2; May 2012.

29. Petya Kasnakova, PhD.Rehabilitation, Rehabilitation of Myofascia Pain Syndrome in Over tension Related diseases; East European Scientific Journal III, 2015; 6-8.

30. Julie Ann Day,Carla Stecco, Antonio Stecco .Application of Fascial Manipulation\& technique in chronic shoulder pain-Anatomical basis and clinical implications; Journal of Bodywork and Movement Therapies;2009;13;128-135.

31. Mariana Ruiz-Sáez, César Fernández-de-las-Peñas, Cleofás Rodrı'guez Blanco, Raquel Martı́nez-Segura et al. Changes in pressure Pain sensitivity in latent Myofascial trigger point in the upper trapezius muscle after a cervical spine stimulation; J Manipulative Physiology Ther 2007;30:578-583 
32. Tim Speicher, David O. Draper. Top 10 Positional-Release Therapy Techniques to Break the Chain of Pain, Part 1; Human Kinetics · ATT 11(5); September 2006; 60-62.

33. Fryer G. Muscle energy technique: An evidence-informed approach. Int J Osteopath Med. 2011; 14(1):3-9

34. L. DiLorenzo M, Traballesi, D. Morelli, A. Pomp, S. Brunelli, M. G. Buzzi et al. Hemi paretic Shoulder Pain Syndrome Treated with Deep Dry Needling During Early Rehabilitation: A Prospective, Open-Label, Randomized Investigation; Journal of Musculoskeletal Pain, Vol. 12(2); 2004;25-34.

35. MarcoBarbero, Corrad, Cescon, Andrea, Tettamani, Vittorio Leggero, FionMacmillan, Fiona Coutts et al. Myofascial trigger points and innervations zone locations in upper trapezius muscles; BMC Musculoskeletal Disorders 2013; 14:179; 2-9.

36. Anil KumarJain, Dan S. Heffez, Bruce M. Carruthers Frances Y.-K. Leun, Marjorie I. van de Sande et al. Fibromyalgia Syndrome: Canadian Clinical Working Case Definition, Diagnostic and Treatment Protocols-A Consensus Document; Journal of Musculoskeletal Pain; Vol. 11, No. 4, 2003; 3-107.

37. Lilian Albert Zaky, Bassem El Nahass and Aly Mohamed El Zawahry. Myofascial Trigger Points Pressure Release versus Exercises Therapy in the Treatment of Chronic Cervical Myofascial Pain Dysfunction Syndrome; Bull. Fac. Ph. Th. Cairo Univ., Vol. 15, No. (1) Jan. 2010; 83-92.

38. D. Jankovic and A.Van Zundert. The Frozen Shoulder Syndrome Description of a new technique and five case reports using the subscapular nerve block and subscapularis trigger point infiltration; Acta Anaesth. Belg., 2006, 57; 137-143.

39. Francisco Garc1'a-Muro, Angel L. Rodri'guez-Ferna'ndez, Ángel Herrero-de-Lucas. Treatment of myofascial pain in the shoulder with Kinesio Taping. A case report; Manual Therapy; 2009; $1-4$.

40. Abeer A. Yamany and Samir Elsaid Salim Efficacy of Low Level Laser Therapy for Myofascial Trigger Points of Shoulder Pain; World Applied Sciences Journal,12 (6); 2011; 758-764.

41. Sibby, George Mathew, Narasimman, Kavitha Vishal. Effectiveness of Integrated Neuromuscular Inhibitory Technique and Laser with Stretching In the Treatment of Upper Trapezius Trigger Points; Journal of Exercise Science and Physiotherapy, Vol. 5, No. 2;2009;115-121.

42. Narayan, Anupama, Jagga, Vinay. Efficacy of Muscle Energy Technique Functional Ability of Shoulder in Adhesive Capsulitis; Journal of Exercise Science and Physiotherapy, Vol. 10, No. 2:2014; 72-76.

43. Jerrold M, Gorski, MD and Lawrence H.Schwartz. Shoulder Impingement Presenting as Neck Pain; the Journal of Bone \& Joint Surgery JBJS.org Vol 85- Number 4 April 2003; 635-638.

44. Hooten WM, Timming R, Belgrade M, Gaul J, Goertz M, Haake B. Institute for Clinical Systems Improvement. Assessment and Management of Chronic Pain. November 2013. 